\title{
Modeling of photons trapping effect on the performance of HPT-LED Optoelectronic Integrated Device (OEID)
}

\author{
Sh.M. Eladl \\ Rad. Eng. Dept., NCRRT, P.O. Box 29, Nasr City, Atomic Energy Authority, Egypt \\ E-mail: Shaban_45@yahoo.com
}

\begin{abstract}
The effect of photons trapped at the LED side due to total internal reflection on the transient behavior of an Optoelectronic Integrated Device (OEID) is considered in this paper. The device is composed of a Heterojunction Phototransistor (HPT) and a Light Emitting Diode (LED). The expressions describing the transient response of the output photons flux, the rise time, and the output derivative are derived. The effect of the various device parameters on the transient response is outlined. The results show that the transient response of these types of devices is strongly dependent on the ratio of these trapped photons in the LED part. Also the device under consideration can be changed from switching mode to the amplification mode, if the fractions of trapped photons exceed a specified value. This type of the model can be exploited as an optical amplifier, optical switching device and other applications.
\end{abstract}

Keywords: optoelectronic integrated device, heterojunction phototransistor, light emitting diode.

Manuscript received 08.02.09; revised manuscript received 05.04.09; accepted for publication 14.05.09; published online 15.05.09.

\section{Introduction}

Optoelectronic Integrated Devices (OEIDs) have received more attention nowadays due to their potential applications in various areas, such as optical amplifications, switching, and communications [1-5]. This type of devices is still demanded for the evolution of optical communication and optical signal processing because the detecting part possesses the feature of a transistor in most cases. One or more OEIDs can function as bistable optical switches, optical inverters, AND, NAND, and NOR gates. Other structure is suitable for RCE [7], making these devices ideal for WDM optical interconnects. One type of OEIDs consists of a Heterojunction Phototransistor (HPT) that is vertically integrated with a Light Emitting Diode (LED). The input light illuminates the phototransistor, and it is converted into photoexcited carriers that leave the HPT part and are injected into the LED active region. Due to the wide-gap confinement layers, most of these carriers recombine there leading to the emission of an intensified light from the LED side. Fraction of this emitted light is trapped in the LED will be reabsorbed by the carriers in the LED active layer.

A stability testing of a new version of OEIDs was developed in [8]. The testing demonstrates that its optical gain is stable as long as the value of the optical feedback is maintained below the threshold value, while exhibits instability for values of optical feedback, which are greater or equal to this threshold value. Recently, this type of structure has been exploited for optical upconversion devices that convert input $1.5 \mu \mathrm{m}$ light to output $0.87 \mu \mathrm{m}$ light with a built-in gain mechanism [9]. Incoming $1.5 \mu \mathrm{m}$ optical radiation is absorbed by the HPT, generating an amplified photocurrent. The resultant photocurrent drives the LED that emits at $0.87 \mu \mathrm{m}$, which could be detected by a conventional silicon charge-coupled device.

More recently [10], a numerical analysis for dynamic response of a coupled periodic multi-quantum wells heterojunction phototransistor (CP-MQW HPT) integrated over a strained quantum well laser diode was developed. It was observed that the possibility of operation of the developed device in amplification and switching modes was also available as similar to conventional types. In this paper, a detailed investigation of the transient behavior of OEID is presented taking into account the photon mechanism resulted from the trapped photons at the LED side due to total internal reflection process.

The device characteristics under ionizing irradiation are investigated based on the equivalent circuit of the constituent devices and the optical feedback inside the device by Ref. [11]. The switching voltage of this type 
device was increased with the ionizing radiation. The purpose of the current paper is to use a simple method to analyze the transient characteristics of optoelectronic integrated devices, the effect of the photons trapping at the LED side due to internal reflection on the transient behavior of these devices is taken into consideration. The paper is organized as follows: formulation of the specified parameters that describe the transient response, derivative, and rise time of OEID is presented in Section 2. The generated curves resulting are outlined and discussed in Section 3. Finally, conclusion of the work is discussed in Section 4.

\section{Theoretical analysis}

The block diagram of the considered optoelectronic integrated device with optical feedback is shown in Fig. 1a. The output photon flux can be expressed as

$\phi_{\text {out }}=\frac{T(1-\delta) \eta_{\text {int }} J}{q\left[1-\delta \eta_{\text {int }}\right]}$

Where $J$ is the excited photocurrent inside the OEID and has two components, namely: the first one is due to the direct light incident on HPT from the external source, the second one is due to the light back inside the device from the LED to HPT, $\delta$ represents the ratio of photons that trapped within the LED active region due to the internal reflection at the LED interface, $T$ is the transmission coefficient at the LED interface, $q$ is the electric charge, while $\eta_{\text {int }}$ is the LED internal quantum efficiency.

The excited photocurrent density inside the device, if the optical feedback is considered, can be expressed as [3]:

$$
J(\omega)=\frac{q g(\omega) \phi_{\text {in }}(\omega)}{1-k(\omega) g(\omega) \eta(\omega)},
$$

where $g(\omega)$ and $\eta(\omega)$ denote the frequency response of the optical conversion gain of HPT and the external quantum efficiency of the LED, respectively, and $k(\omega)$, the ratio of the photons which reach the HPT to those emitted by the LED that is called as optical feedback and assumed to be constant, $k(\omega)=k$. This optical feedback is assumed to be positive one, because it is added to the main input light with no phase changing due to the small time delay concerning it compared with the forward path time delay.

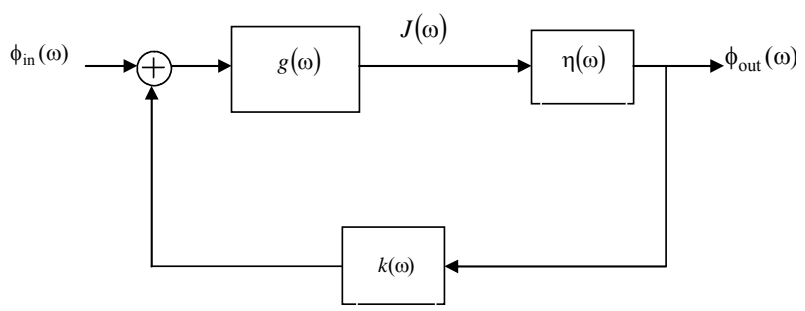

Fig. 1a. Block diagram of OEID with optical feedback.
Using Eqs (1) and (2), the formula for the frequency response of the output photon flux can be expressed as:

$\phi_{\text {out }}(\omega)=\frac{T(1-\delta)}{\left[1-\delta \eta_{\text {int }}(\omega)\right]} \frac{g(\omega) \eta_{\text {int }}(\omega) \phi_{\text {in }}(\omega)}{1-k(\omega) g(\omega) \eta_{\text {int }}(\omega)}$.

When the input light is assumed as a step function in time, the Laplace transform of the photon flux in the case of no optical feedback $(k=0)$ can be obtained as

$$
\frac{\phi_{\text {out }}}{\phi_{\text {in }}}(s)=\frac{T(1-\delta) g_{0} \eta_{\text {int } 0}}{s\left(1+\frac{s}{\omega_{\beta}}\right)\left(1+\frac{s}{\omega_{1}}\right)\left(1-\left(\delta \eta_{\text {int } 0} /\left(1+\frac{s}{\omega_{1}}\right)\right)\right)} .
$$

Where $g_{0}=\beta_{0} \eta h_{0}$ denotes the conversion gain of the HPT in the low frequency regime, and $\beta_{0}$ and $\eta h_{0}$ are the current gain and the quantum efficiency of the HPT in the low frequency regime, and $\omega_{\beta}$ is the beta cut-off frequency. $\omega_{1}$ is the cut-off frequency of the LED where $\omega_{1}^{-1}=\tau_{0}$ is the minority carrier lifetime.

The time response of $\frac{\phi_{\text {out }}}{\phi_{\text {in }}}$ for the optoelectronic integrated devices can be obtained from the inverse Laplace of Eq. (4) as

$\frac{\phi_{\text {out }}}{\phi_{\text {in }}}(t)=L^{-1}\left\{\frac{T(1-\delta) g_{0} \eta_{\text {int } 0}}{s\left(1+\frac{s}{\omega_{\beta}}\right)\left(1+\frac{s}{\omega_{1}}\right)\left(1-\left(\delta \eta_{\text {int } 0}\left(1+\frac{s}{\omega_{1}}\right)\right)\right)}\right\}$

Thus, the time response of $\frac{\phi_{\text {out }}}{\phi_{\text {in }}}$ can be obtained as: $\frac{\phi_{\text {out }}}{\phi_{\text {in }}}(t)=\left[T(1-\delta) \eta_{\text {int } 0} g_{0} \omega_{1} \omega_{\beta}\right] \times$

$\times\left\{\begin{array}{l}\frac{1}{\omega_{1} \omega_{\beta}\left(1-\delta \eta_{\text {int } 0}\right)}-\frac{1}{\omega_{\beta}\left(\omega_{1}-\delta \eta_{\text {int } 0} \omega_{1}+\omega_{\beta}\right)} e^{-\omega_{\beta} t} \\ -\frac{1}{\omega_{1}\left(1-\delta \eta_{\text {int } 0}\right)\left(\omega_{1}-\omega_{\beta}-\delta \eta_{\text {int } 0} \omega_{1}\right)} e^{-\omega_{1}\left(1-\delta \eta_{\text {int } 0}\right) t}\end{array}\right\}$.

If $\omega_{1}>\omega_{\beta}$, the above equation can be reduced to the following form

$\frac{\phi_{\text {out }}}{\phi_{\text {in }}}(t)=\frac{T(1-\delta) \eta_{\text {int } 0} g_{0}}{1-\delta \eta_{\text {int } 0}}\left[1-e^{-\omega_{\beta} t}\right]$.

The derivative of $\frac{\phi_{\text {out }}}{\phi_{\text {in }}}$ for the OEID with respect to time is expressed by

$$
\begin{aligned}
& \frac{d}{d t} \frac{\phi_{\text {out }}}{\phi_{\text {in }}}(t)=\frac{T(1-\delta) \eta_{\text {int } 0} g_{0} \omega_{1} \omega_{\beta}}{\left(\omega_{1}-\omega_{\beta}-\delta \eta_{\text {int } 0} \omega_{1}\right)} \times \\
& \times\left\{\frac{\left(\omega_{1}-\delta \eta_{\text {int } 0} \omega_{1}\right)}{\omega_{1}\left(1-\delta \eta_{\text {int } 0}\right)} e^{-\omega_{1}\left(1-\delta \eta_{\text {int } 0}\right) t}-e^{-\omega_{\beta} t}\right\} .
\end{aligned}
$$


The above expression describes how fast the output photons change with time. Using the approximation $\omega_{1} \gg \omega_{\beta}$ will yield to

$$
\frac{d}{d t} \frac{\phi_{\text {out }}}{\phi_{\text {in }}}(t)=\frac{T(1-\delta) \eta_{\text {int } 0} g_{0}}{\left(1-\delta \eta_{\text {int } 0}\right)}\left\{e^{-\omega_{\beta} t}\right\} .
$$

The rise time of $\frac{\phi_{\text {out }}}{\phi_{\text {in }}}$ for optoelectronic integrated devices is defined as the time required for $\frac{\phi_{\text {out }}}{\phi_{\text {in }}}(t)$ to rise to 0.9 of its final value, by solving Eq. (6). The rise time can be given as

$T_{A}=\frac{1}{\omega_{\beta}} \ln \left[\frac{\frac{T(1-\delta) \eta_{\text {int } 0} g_{0}}{1-\delta \eta_{\text {int } 0}}-0.9 \phi_{a}}{\frac{T(1-\delta) \eta_{\text {int } 0} g_{0}}{1-\delta \eta_{\text {int } 0}}}\right]$.

When

$\phi_{a}=\frac{T(1-\delta) \eta_{\text {int } 0} g_{0}}{1-\delta \eta_{\text {int } 0}}$, then $T_{A}=\frac{2.3}{\omega_{\beta}}$.

If the optical feedback inside the device is considered, the following equation will be valid:

$\frac{\phi_{\text {out }}}{\phi_{\text {in }}}(s)=\frac{T(1-\delta) g_{0} \eta_{\text {int } 0}}{Z(s)}$,

where

$$
\begin{aligned}
& Z(s)=s\left(1+\frac{s}{\omega_{\beta}}\right)\left(1+\frac{s}{\omega_{1}}\right)\left(1-\frac{\delta \eta_{\text {int } 0}}{\left(1+\frac{s}{\omega_{1}}\right)}\right) \times \\
& \times\left[1-\frac{k g_{0} \eta_{\text {int } 0}}{\left(1+\frac{s}{\omega_{\beta}}\right)\left(1+\frac{s}{\omega_{1}}\right)}\right] .
\end{aligned}
$$

Using the approximation $\omega_{1}>>\omega_{\beta}$ will yield to

$$
\begin{aligned}
& \frac{\phi_{\text {out }}}{\phi_{\text {in }}}(t)=\frac{T\left(1-\delta \eta_{\text {int } 0}\right)(1-\delta) \eta_{\text {int } 0} g_{0} \omega_{1} \omega_{\beta}}{\left(1-(1-\delta) k \eta_{\text {int } 0} g_{0}\right)\left(1-\delta \eta_{\text {int } 0}\right)^{2}} \times \\
& \times\left\{1-e^{-\omega_{\beta}\left(1-\frac{k \eta_{\text {int } 0} g_{0}((1-\delta))}{\left(1-\delta \eta_{\text {int } 0}\right)}\right) t}\right\} .
\end{aligned}
$$

The derivative of the output photon flux emerging from OEID with respect to time is expressed by

$$
\begin{aligned}
& \frac{d}{d t} \frac{\phi_{\text {out }}}{\phi_{\text {in }}}(t)=\frac{T(1-\delta) \eta_{\text {int } 0} g_{0}}{\left(1-\delta \eta_{\text {int } 0}\right)\left(1-(1-\delta) k \eta_{\text {int } 0} g_{0}\right)} \times \\
& \times\left\{e^{-\omega_{\beta}\left(1-(1-\delta) k \eta_{\text {int } 0} g_{0}\right) t}\right\} .
\end{aligned}
$$

The rise time of the output photon flux, where the optical feedback is taken into consideration, can be expressed as:

$T_{A}=\frac{1-\delta \eta_{\text {int } 0}}{\omega_{\beta}\left(\left(1-\delta \eta_{\text {int } 0}-(1-\delta) k \eta_{\text {int } 0} g_{0}\right)\right)} \ln [R]$.

Here

$R=\left[\frac{T(1-\delta) \eta_{\text {int } 0} g_{0}-0.9\left(1-\delta \eta_{\text {int } 0}-(1-\delta) k \eta_{\text {int } 0} g_{0}\right) \phi_{a}}{T(1-\delta) \eta_{\text {int } 0} g_{0}}\right]$,

where $\phi_{a}$ is the steady state output photon flux

$\phi_{a}=\frac{T(1-\delta) \eta_{\text {int } 0} g_{0}}{1-\delta \eta_{\text {int } 0}\left(1-(1-\delta) k \eta_{\text {int } 0} g_{0}\right)}$.

Then,

$T_{A}=\frac{2.3\left(1-\delta \eta_{\text {int } 0}\right)}{\omega_{\beta}\left(1-\delta \eta_{\text {int } 0}-(1-\delta) k \eta_{\text {int } 0} g_{0}\right)}$.

\section{Results and discussion}

The device parameters used in the subsequent calculated figures are the same as those used by $\mathrm{Zhu}$ et al. [6], where $\omega_{\beta}=10^{8} \mathrm{~Hz}, \omega_{1}=10^{10} \mathrm{~Hz}, T=0.7, \delta=0.5$, and $\eta_{\text {int } 0} g_{0}=100$. The input light flux $\phi_{\text {in }}$ is assumed to be a step function in time. A complete schematic picture of the proposed OEID is shown in Fig. 1b. Since the same InGaAs active region is used for both HPT and LED, some portion of the same spectral generated light at the LED side traverses back through the cladd and collector regions to be absorbed again at the HPT InGaAs active region causing an optical feedback. The thickness of the cladd and collector regions play a significant role on controlling the magnitude of this optical feedback, the amplitude of the optical feedback is inversely proportional to the thickness of these two regions, while it is directly proportional to the thickness of the two active regions. The type of materials of the active regions has the major effect on the properties of the spectral response for the generated light. The transient response of $\phi_{\text {out }} / \phi_{\text {in }}$ of the OEID in the amplification mode is shown in Fig. 2. It can be seen that $\phi_{\text {out }} / \phi_{\text {in }}$ of the device approaches a definite value, this definite value depends on the optical conversion gain of the HPT, external quantum efficiency of the light emitting diode, the value of the optical feedback within the device, and the value of the trapping factor at the LED side. The value of external quantum efficiency of the LED is much lower than the internal one due to reabsorption and total internal reflection within the LED active region. In order to prevent reabsorption, the layer above the active region has to be with higher band gap than that of the active region to ensure good confinement of photons inside this layer. Also, the device operates in a stable mode called the amplification mode. 


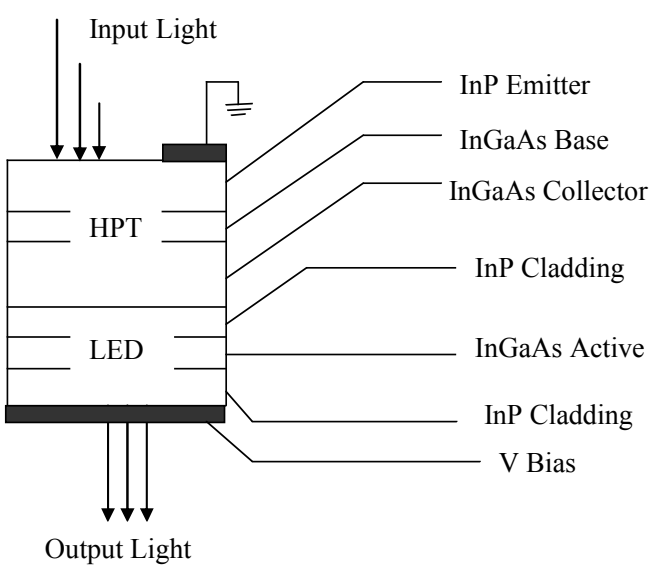

Fig. 1b. Schematic view of the proposed OEID.

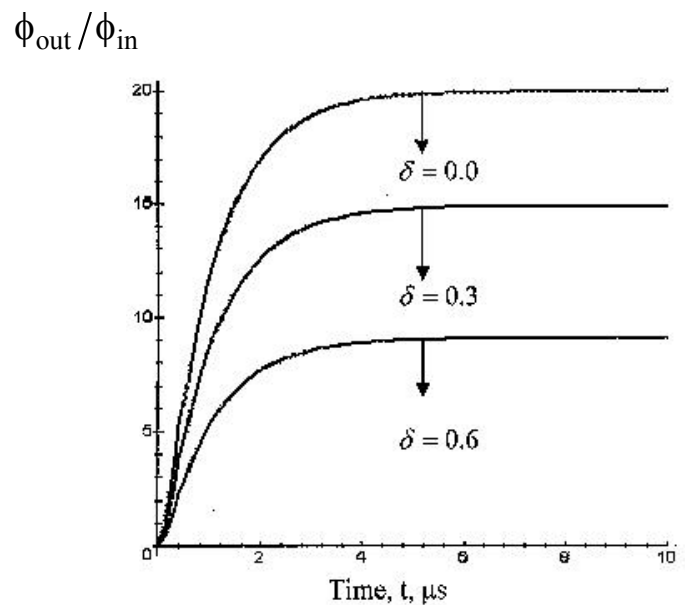

Fig. 2. Amplification mode transient response of $\phi_{\text {out }} / \phi_{\text {in }}$ versus time at different $\delta$ values.

Fig. 3 plots $\frac{d}{d t} \frac{\phi_{\text {out }}}{\phi_{\text {in }}}(t)$ versus time at different values of $\delta$, where the value of this derivative is used to measure the speed of photon flux growth that emerges from the device. The plot exhibits a pronounced maximum value at a certain time, and after that it decays exponentially to a minimum value where the output flux reaches its final value. At any time below this specified value, the derivative increases with time, while the derivative decreases with time above this specified value of time. If the optical feedback within the device is increased, the obtained $\phi_{\text {out }} / \phi_{\text {in }}$ will increase, while this specified value will not be affected or changed by the increase in optical feedback.

Fig. 4 illustrates the decrease in $\phi_{\text {out }} / \phi_{\text {in }}$ with increasing $\delta$. Such decrease is more pronounced in LEDs with a small quantum efficiency in which the output photon flux is smaller. The HPT conversion gain play a major role in obtaining a higher $\phi_{\text {out }} / \phi_{\text {in }}$ value, where the higher optical conversion gain of HPT means

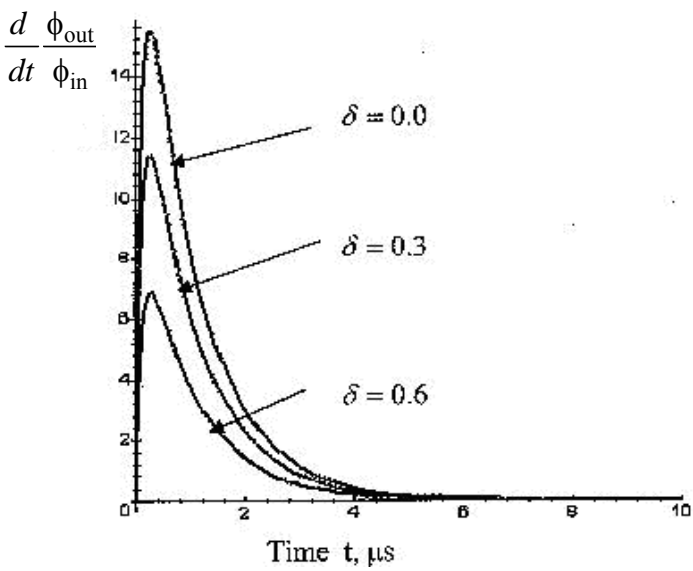

Fig. 3. Derivative of output photon flux with time at different $\delta$ values.

$$
\phi_{\text {out }} / \phi_{\text {in }}
$$

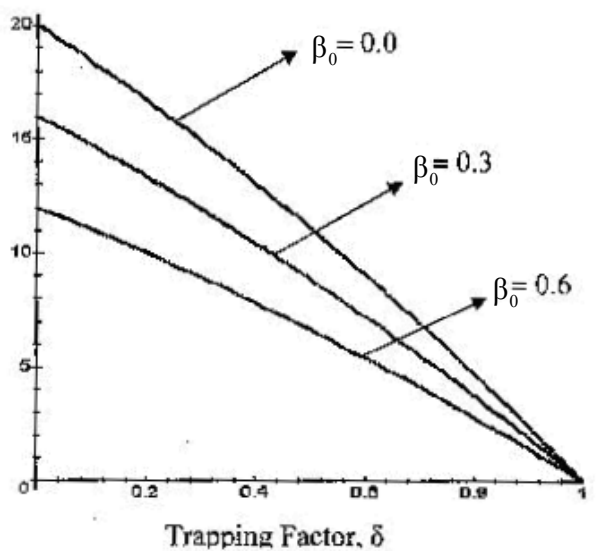

Fig. 4. Dependence of $\phi_{\text {out }} / \phi_{\text {in }}$ on the trapping factor at different $\beta_{0}$ values.

higher current density injected to the LED part and, hence, a higher $\phi_{\text {out }} / \phi_{\text {in }}$ value. Fig. 5 shows the time dependence of $\phi_{\text {out }} / \phi_{\text {in }}$ at different values of $\delta$. This operation mode is the so-called switching mode where $\phi_{\text {out }} / \phi_{\text {in }}$ is increased linearly and exhibits an abrupt change from low current state to high current state, which agree with the switching characteristics. From the figure, the values of $\delta$ limit the switching speed where the device with lower $\delta$ switches earlier than that of higher $\delta$. Faster and higher performance OEID can be achieved using the LED with lower trapping factor. Since $\delta$ represents the fraction of photons propagating outside the critical angle cone of the LED, so it is necessary to decrease its value to ensure maximum output flux of photons through the LED active region. III-V materials have small critical angle cone, therefore the radiation emitted suffers from total internal reflection. To decrease the value of $\delta$, the refractive index of the LED active region has to be chosen with a small value. 


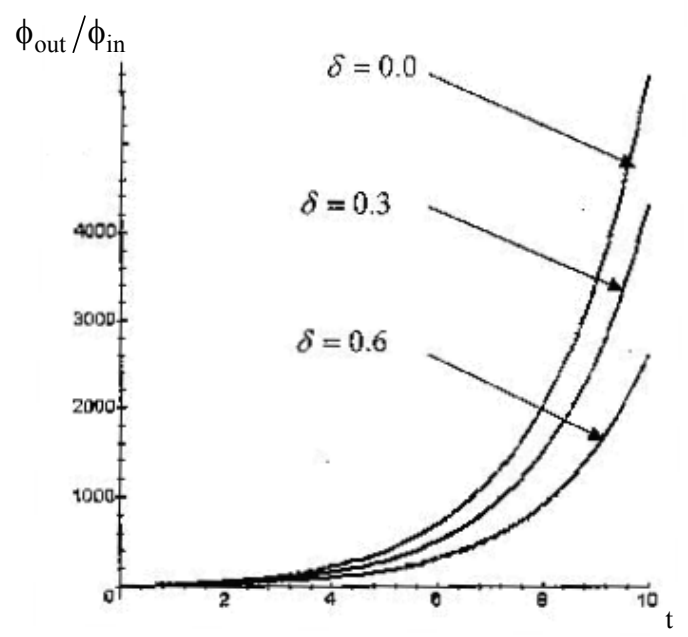

Fig. 5. Switching mode transient response of $\phi_{\text {out }} / \phi_{\text {in }}$ versus time at different $\delta$ values.

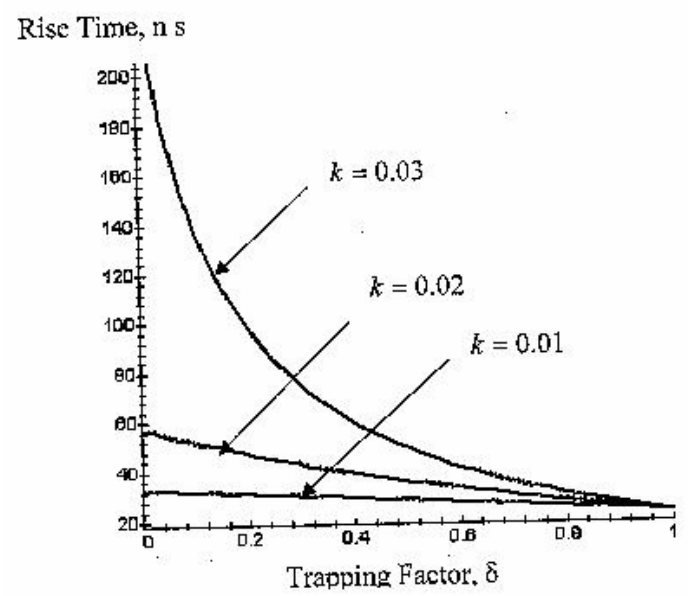

Fig. 6. Rise time versus $\delta$ at different values of optical feedback $k$.

The dependence of the rise time of $\phi_{\text {out }} / \phi_{\text {in }}$ of OEID on the optical feedback coefficient in the amplification mode is shown in Fig. 6. It is clear that by increasing the optical feedback, there is an increase in the rise time due to the increase of the difference between $\phi_{\text {out }} / \phi_{\text {in }}$ in the initial and the final state. The optical feedback is usually weakened in the amplification mode by inserting an absorption layer between the HPT and LED, and thus the rise time in this mode is equal in magnitude as that of the HPT with optical feedback.

\section{Conclusions}

A theoretical model including the effect of photons trapped in the LED side due to total internal reflection on behavior of an Optoelectronic Integrated Device was proposed and used to evaluate its characteristics. Analytical formulas for the transient response, derivative, and rise time were derived. The results show that photon trapping within the LED region strongly influences the device gain and switching speed. Optical feedback influences crucially functions and operation modes where the lower values of optical feedback allow the device to operate in amplification mode, while the higher values to operate in the switching mode. The obtained expressions can be used for the optimization of the device performance.

\section{References}

1. S.A. Feld, F.R. Beyette, Jr., M.J. Hafich, H.Y. Lee, G.Y. Robinson, and C.W. Wilmsen, Electrical and optical feedback in an InGaAs/InP Light Amplifying Optical Switch (LAOS) // IEEE Trans. Electron Device 38, No. 11, p. 2452-2458 (1991).

2. A. Vahid, S. Noda, A. Sasaki, Analysis for relative intensity noise of optoelectronic integrated device by heterojunction phototransistor and laser diode // Solid State Electron. 41, p. 465-471 (1997).

3. Susumu Noda, Toru Takayama, Kimitaka Shibata, and Akio Sasaki, High gain and very sensitive photonic switching device by integration of heterojunction and laser diode // IEEE Trans. Electron Devices 39, No. 2, p. 309-311 (1992).

4. S. Noda, K. Yasuhiro, and A. Sasaki, Optoelectronic integrated tristable device with optically controlled set and reset functions // $J$. Quantum Electron. 31, No. 8, p. 1465-1473 (1995).

5. Satyabrata Jit and B.B. Paul, A new Optoelectronic Integrated Device for Light Amplifying Optical Switch (LAOS) // IEEE Trans. Electron Devices 48, No. 12, p. 2732-2739 (2001).

6. Yu Zhu, Susumu Noda, and Akio Sasaki, Theoretical analysis of transient behavior of optoelectronic integrated devices // IEEE Trans. Electron Devices 42, No. 4, p. 646-648 (1995).

7. M.S. Unlü, S. Strite, A. Salvador, A.L. Demirel, and H. Morkoc, Wavelength discriminating optical switch // IEEE Photon. Tech. Lett. 3, No. 12, p. 1126-1129 (1991).

8. M.B. El Mashade, M. Ashry, Sh.M. Eladl, M.S. Rageh, Performance analysis and stability testing of a new structure of optoelectronic integrated device // Microelectron. J. 35, No. 7, p. 585-589 (2004).

9. H. Luo, D. Ban, H.C. Liu et al., Optical upconverter with integrated heterojunction phototransistor and light-emitting diode // Appl. Phys. Lett. 88, 073501 (2006).

10. E. Darabi, V. Ahmadi, and K. Mirabbaszadeh, Numerical analysis of an optoelectronic integrated device composed of coupled periodic MQW phototransistor and strained-QW laser diode // Solid-State Electron. 50, No. 3, p. $473-479$ (2006).

11. Sh.M. Eladl, Modeling of ionizing radiation effect on optoelectronic-integrated devices (OEIDs) // Microelectron. J. 40, No. 1, p. 193-196 (2009). 